\title{
Nonlinear Development and Secondary Instability of Traveling Crossflow Vortices
}

\author{
Fei Li*, Meelan M. Choudhari ${ }^{*}$ \\ NASA Langley Research Center, Hampton, VA 23681 \\ Lian Duan ${ }^{+}$ \\ Missouri University of Science and Technology, Rolla, MO 65409 \\ Chau-Lyan Chang ${ }^{\times}$ \\ NASA Langley Research Center, Hampton, VA 23681
}

\begin{abstract}
Transition research under NASA's Aeronautical Sciences Project seeks to develop a validated set of variable fidelity prediction tools with known strengths and limitations, so as to enable "sufficiently" accurate transition prediction and practical transition control for future vehicle concepts. This paper builds upon prior effort targeting the laminar breakdown mechanisms associated with stationary crossflow instability over a swept-wing configuration relevant to subsonic aircraft with laminar flow technology. Specifically, transition via secondary instability of traveling crossflow modes is investigated as an alternate scenario for transition. Results show that, for the parameter range investigated herein, secondary instability of traveling crossflow modes becomes insignificant in relation to the secondary instability of the stationary modes when the relative initial amplitudes of the traveling crossflow instability are lower than those of the stationary modes by approximately two orders of magnitudes or more. Linear growth predictions based on the secondary instability theory are found to agree well with those based on PSE and DNS, with the most significant discrepancies being limited to spatial regions of relatively weak secondary growth, i.e., regions where the primary disturbance amplitudes are smaller in comparison to its peak amplitude. Nonlinear effects on secondary instability evolution is also investigated and found to be initially stabilizing, prior to breakdown.
\end{abstract}

\section{Nomenclature}

A $\quad=$ amplitude of crossflow instability mode or secondary instability mode at given chordwise location defined as ratio of chordwise perturbation velocity to freestream velocity

$A_{\text {init }} \quad=$ initial amplitude, i.e., amplitude at location where initial perturbation is imposed in computation

AoA $\quad=$ angle of attack

$c=$ wing chord length measured in direction perpendicular to leading edge

$f \quad=$ frequency of instability oscillations

$M \quad=$ freestream Mach number

$m \quad=$ Fourier index of frequency mode

$N=$ logarithmic amplitude ratio, i.e., $\mathrm{N}$-factor of linear crossflow instability or secondary instability

$n=$ Fourier index of spanwise mode

$R e_{c} \quad=$ Reynolds number based on streamwise chord length

$t \quad=$ time

$U_{\infty} \quad=$ freestream velocity

$u \quad=$ chordwise perturbation velocity

$x=$ chordwise coordinate in direction perpendicular to leading edge

$y=$ wall-normal coordinate

\footnotetext{
* Aerospace Technologist, fei.li@nasa.gov

\# Aerospace Technologist, meelan.m.choudhari@nasa.gov, Associate Fellow, AIAA.

+ Assistant Professor, duanl@mst.edu, Senior Member, AIAA.

× Aerospace Technologist, chau-lyan.chang@nasa.gov, Senior Member, AIAA.
} 
$z=$ spanwise coordinate in direction parallel to leading edge

$\lambda_{z}=$ spanwise wavelength

LSIT = linear secondary instability theory

$P S E \quad=$ parabolized stability equations

NPSE $=$ nonlinear parabolized stability equations

DNS = direct numerical simulations

TS $=$ Tollmien-Schlichting

LASTRAC $=$ Langley Stability and Transition Analysis Codes

\section{Introduction}

The economic and environmental benefits of laminar flow technology via reduced fuel burn of subsonic and supersonic aircraft cannot be realized without minimizing the uncertainty in drag prediction in general and transition prediction in particular. Transition research under NASA's Aeronautical Sciences Project seeks to develop a validated set of variable fidelity prediction tools with known strengths and limitations, so as to enable sufficiently accurate transition prediction and practical transition control for future vehicle concepts. The purpose of the present research is to investigate transition mechanisms involving the breakdown of traveling crossflow vortices, which have received less attention than their stationary counterpart.

In general, a swept wing boundary layer can be susceptible to various types of primary instabilities such as attachment line instability, ${ }^{1}$ stationary and traveling crossflow modes, and Tollmien-Schlichting (TS) waves. Regardless of which mode dominates the primary amplification stage, the other modes could play a role during the nonlinear stage and hence, influence the onset of transition. Although, in cases of practical interest, the breakdown of stationary crossflow vortices appears to be the more likely route to transition, ${ }^{2-6}$ other scenarios involving a more significant role for the traveling crossflow instability ${ }^{7-10}$ may become relevant under less common circumstances. These may happen either when the initial amplitudes of traveling modes become sufficiently large as a result of higher amplitude freestream disturbances or when the surface has been carefully polished to minimize the initial amplitudes of stationary crossflow modes. Wassermann and Klocker ${ }^{8}$ studied the nonlinear saturation of traveling crossflow vortices and the associated developments of secondary instability for a flat plate configuration subject to a favorable pressure gradient. Among their findings are that the finite amplitude traveling crossflow vortices form stronger internal shear layers which are much closer to the wall than their stationary counterpart and that the secondary instability is dominated by the Z-mode type, a terminology first introduced in Ref. 2. Choudhari et al. ${ }^{9}$ used NPSE and LSIT to analyze the traveling crossflow vortices and their secondary instability on the same configuration as in the present paper and found similar behavior for the finite amplitude crossflow vortices, but also picked up the Y-mode ${ }^{2}$ secondary instability, which becomes dominant when the amplitude of the primary crossflow vortex is large. Down and White ${ }^{10}$ studied the effect of freestream turbulence on the development of crossflow disturbances in a recent experiment and showed that increasing freestream turbulent levels promote the traveling crossflow vortices. Their paper also included a thorough review of the previous work on this topic.

In a practical setting, the investigation of nonlinear breakdown scenarios associated with a mixed evolution of stationary and traveling crossflow modes is perhaps more important. However, as a prelude to the more complex scenario, the secondary instability of traveling crossflow modes alone is studied herein to obtain useful insights regarding their breakdown. Results presented in a previous paper ${ }^{9}$ showed that the traveling crossflow modes saturate at much lower amplitudes in comparison to the stationary modes and, yet, the growth of secondary instabilities of the traveling crossflow vortices can be at least as strong as the secondary modes of the stationary crossflow modes. The work presented herein extends that effort to address the important issue of what threshold initial amplitudes are required for the traveling vortices to sustain a significant amplification of secondary instabilities. Additionally, the predictions based on the secondary instability theory are compared with direct numerical simulations (DNS) with controlled initial disturbances.

The paper is organized as follows. Section II provides a brief background on the secondary instability theory for traveling crossflow instabilities in a swept wing boundary layer. For a more complete description of underlying methodology, the reader is referred to Ref. 11. The flow configuration of interest in this paper is outlined in Section III, along with the computational codes used in the analysis. The computational results are discussed in Section IV, followed by concluding remarks in Section V. 


\section{Secondary Instability}

Secondary instability is an instability mechanism of the primary instability. Examples of primary instabilities in swept wing boundary layers include Tollmien-Schlichting waves, Görtler vortices, and crossflow vortices. The initial growth of a primary instability of infinitesimal amplitude is usually exponential and, as the amplitude increases downstream, nonlinear effects often lead to a quasi-saturation behavior. In strictly parallel flows such as a plane Poiseuille flow, the primary amplitude reaches a finite constant value. In many other cases such as external boundary-layer flows, however, the primary wave amplitude continues to evolve slowly as a result of the slow streamwise variations in the base flow.

The quasi-saturated, finite amplitude primary wave can become susceptible to an instability of its own, namely, the secondary instability. Many examples of primary waves are time-independent when viewed in a proper inertial frame of reference and are periodic in at least one spatial direction. Görtler and stationary crossflow vortices are time-independent in a fixed frame of reference and are periodic in the spanwise direction in a boundary layer that is invariant in the spanwise direction. Traveling crossflow vortices in such boundary layers are also periodic in the spanwise direction and appear stationary when the observer travels with the spanwise phase velocity of the vortices.

A local secondary instability analysis becomes possible when the slowly varying base flow is approximated as locally constant along the direction of slow mean flow evolution, because the coefficients of the perturbation equation are now locally independent of that direction. The stationary and periodic nature of the primary waves greatly facilitates the analysis of secondary instabilities. If the flow field with a saturated primary wave is used as the base flow on which an infinitesimal perturbation is introduced, then the resulting linearized equations governing the evolution of the perturbation take the form of a set of linear partial differential equations with periodic coefficients in one of the directions. For Görtler and crossflow vortices, the periodic direction is along the span of an airfoil.

When the primary instability corresponds to crossflow vortices, the base flow is slowly varying along the vortex axis but periodic in the spanwise direction with the same period as the spanwise wavelength of the primary crossflow vortex. These two directions are, in general, not orthogonal and the problem is best solved in a nonorthogonal coordinate system. The form of the secondary instability equations in a non-orthogonal coordinate system was derived in the context of stationary crossflow instability by Li and Choudhari. ${ }^{11}$ The same framework is applied in this paper to perform a spatial secondary instability analysis for traveling crossflow modes. An extra complication arising in this case is that the base flow itself is unsteady. To circumvent the issue of temporal variations in the base flow, the problem is analyzed in an inertial frame of reference traveling with the spanwise phase velocity of the crossflow vortex, which makes the base flow independent of time as described by Malik et al. ${ }^{12}$

The system of secondary instability equations is subject to boundary conditions of vanishing perturbation quantities at the wall and freestream boundaries. The spanwise boundary conditions are not explicitly specified and they come in as part of the admissible solutions. The main difference between a stability equation governing the secondary instability and that governing the primary instability is that the coefficients of former are functions of two spatial variables instead of just one in the case of the latter. Thus, the resulting eigenvalue problem is also referred to as a $2 \mathrm{D}$ eigenvalue problem. ${ }^{13}$ Since the base flow for the secondary instability riding on crossflow vortices is periodic in the spanwise direction as discussed above, the coefficients of the secondary instability equations are also periodic. Therefore, a class of solutions for the $2 \mathrm{D}$ eigenvalue problem is given by the well-known Floquet theory. ${ }^{14}$ However, in this paper, we are only interested in those solutions that are periodic in the spanwise direction with the same period as that of the base flow.

\section{Flow Configuration and Analysis Codes}

The flow configuration employed in the present study corresponds to the laminar flow airfoil TAMU-003T75 (v.90) described by Belisle et al. ${ }^{15}$ The 9.3 percent thick, 30-degree swept wing is designed to achieve natural laminar flow over approximately 60 and 50 percent of the suction and pressure surfaces, respectively, at the design condition of $M=0.75$, AoA $=0$ deg., and $R e_{c} \approx 17$ million. Here, the non-dimensional parameters $M$ and $\operatorname{Re}_{c}$ are based on the freestream speed, a streamwise chord length of $12 \mathrm{ft}$, and a freestream temperature of $390 \mathrm{deg}$. R, corresponding to a flight altitude of 40,000 ft. Design constraints for the wing included (i) a lift coefficient that is typical of subsonic transports, and (ii) a wing thickness distribution that is suitable for a mid-size business jet. Both 
Tollmien-Schlichting and crossflow instabilities are sufficiently weak at the design angle of attack, so that natural laminar flow should be achievable over a significant portion of the airfoil surface on both suction $(0<x / c<0.6)$ and pressure $(0<x / c<0.5)$ sides without any external means of boundary layer control. In the present research, we focus on the off design condition corresponding to an angle of incidence equal to - 1 degree. At this condition, an even stronger crossflow instability than the design condition is known to exist along the suction side. ${ }^{15}$

For the present work, the mean boundary-layer flow over the suction surface of the airfoil is computed with a boundary layer solver ${ }^{16}$ by using the infinite span approximation, in conjunction with the inviscid surface pressure distribution derived from an Euler solution under free flight conditions. ${ }^{17}$ Linear and nonlinear development of the instability modes is computed using parabolized stability equations (PSE) as implemented in the Langley Stability and Transition Analysis Codes (LASTRAC). ${ }^{18}$ The growth of high frequency secondary instability modes supported by the finite amplitude stationary crossflow vortex is analyzed in a manner similar to the classical linear stability analysis of swept wing boundary layers. Details of the computational methodology employed for linear secondary instability analysis (LSIT) may be obtained from Ref. 11, and the essential modifications for the traveling mode case are mentioned in Ref. 9 and Section II above. DNS computations are also carried out for both finite amplitude traveling crossflow vortices and their secondary instability. Details of the DNS method used in the analysis can be found in Ref. 19 and 20. The main difference between the primary and secondary stability analyses is that the basic state for the secondary modes (i.e., the mean boundary layer flow modified by the primary crossflow mode) varies in both surface normal and spanwise directions and, hence, the instability characteristics of the secondary modes must be analyzed using a planar, partial differential equation based eigenvalue problem, rather than as an ordinary differential equation based eigenvalue problem for the classical analysis. The selection of grid and other aspects of the numerical solution were based on extensive experience with similar class of flows ${ }^{2,9,11,21-24}$ and checks were made to ensure that the impact of variations with respect to those choices was negligible.

\section{Results}

The computational results for the nonlinear development of traveling crossflow vortices and the associated linear growths of secondary instabilities are presented in Subsections A and B below.

\section{A. Nonlinear Evolution of a Traveling Crossflow Mode}

A comparison of the nonlinear amplification characteristics for stationary and traveling crossflow modes with a fixed spanwise wavelength of $\lambda_{z}=8 \mathrm{~mm}$ was presented in Ref. 9. That analysis is expanded herein by considering a broader range of spanwise wavelengths. To allow selection of the most relevant frequency-wavenumber combinations for these nonlinear calculations, linear $\mathrm{N}$-factor computations for the traveling crossflow instability were carried out with linear PSE. This selection process is based on the same premise as the $\mathrm{e}^{\mathrm{N}}$ method for transition prediction, namely that transition is likely to be caused by the instability mode that first achieves a linear amplification ratio corresponding to a specified value of $\mathrm{N}$. This mode may or may not correspond to the mode that achieves the highest overall $\mathrm{N}$-factor over the chord of the airfoil. Based on the abovementioned criterion, traveling crossflow instability modes with spanwise wavelengths of 6,8 and $10 \mathrm{~mm}$ and a frequency of 1,500 Hz were found to reach $\mathrm{N}=10$ before most other traveling modes and, hence, were selected for nonlinear analysis in this paper. For $\lambda_{z}=12 \mathrm{~mm}$, the mode with a lower frequency of $1,225 \mathrm{~Hz}$ reaches $\mathrm{N}=10 \mathrm{first}$; however, its $\mathrm{N}$-factor value of 10 is only slightly higher than that of the traveling crossflow mode with a $1,500 \mathrm{~Hz}$ mode. Therefore, to enable consistent comparisons across different wavelengths, the $1,500 \mathrm{~Hz}$ mode is analyzed in this paper even for the $\lambda_{z}=12 \mathrm{~mm}$ wave.

Figure 1a shows the linear $\mathrm{N}$-factor curves for crossflow instability modes with $\lambda_{z}=8 \mathrm{~mm}$ for selected frequencies ranging from 0 to $2,250 \mathrm{~Hz}$. As expected, the most amplified traveling mode achieves higher $\mathrm{N}$-factors than the stationary mode and, more importantly, the traveling mode with $f=1,500 \mathrm{~Hz}$ reaches $\mathrm{N}=10$ within nearly half the distance it takes for the stationary crossflow mode $(f=0)$ to reach the same $\mathrm{N}$-factor value. This indicates the need to study the breakdown of traveling waves to obtain a more complete picture of crossflow-dominated transition, especially when the relative initial amplitudes of the traveling crossflow modes are not sufficiently small in comparison with those of the stationary modes.

Figure $1 \mathrm{~b}$ shows $\mathrm{N}$-factor curves for various spanwise wavelengths at $1,500 \mathrm{~Hz}$; and the $\lambda_{2}=6 \mathrm{~mm}$ wave reaches an $\mathrm{N}$-factor of 10 before the longer waves, even though the overall maximum $\mathrm{N}$-factor is reached by the $12 \mathrm{~mm}$ mode. 
Comparisons between nonlinear developments of traveling and stationary vortices were presented by Choudhari et al. ${ }^{9}$ Since this is an important component of this analysis, showing characteristic differences between the two types of crossflow vortices, the essential results are reproduced here. Figure 2a shows comparisons of the nonlinear development of stationary and traveling crossflow vortices of the same spanwise wavelength $\left(\lambda_{z}=8 \mathrm{~mm}\right)$ and the same initial amplitude $\left(A_{\text {init }}=1 \times 10^{-5}\right)$. This particular spanwise wave length was chosen because the linear and nonlinear PSE computations by $\mathrm{Li}$ et al ${ }^{23}$ had indicated the crossflow vortex with $\lambda_{z}=8 \mathrm{~mm}$ to be one of the most likely stationary modes to cause transition. The essential results of Ref. 9 are that the traveling crossflow vortices saturate at lower fundamental amplitudes than the stationary vortices, approximately just one half of the saturation amplitude of the latter, while the peak mean flow correction amplitudes for both stationary and traveling modes reach comparable levels. This comparison between the nonlinear evolution of isolated traveling and stationary crossflow disturbances is very similar to that reported by Malik et al. ${ }^{24}$ for a different airfoil configuration and to that by Wassermann and $\mathrm{Kloker}^{8}$ for a flat plate subject to a favorable pressure gradient. Therefore, these findings appear to be more general and not restricted to just one specific case.

Modal amplitudes of traveling crossflow vortices of $1,500 \mathrm{~Hz}$ analyzed in Ref. 9 are shown in Figure $2 \mathrm{~b}$ for initial amplitudes of $2.5 \times 10^{-6}, 5.0 \times 10^{-6}, 7.5 \times 10^{-6}$ and $10^{-5}$. Amplitudes of the mean flow correction and the first harmonic are also shown for the case of the smallest initial amplitude. The general features of oscillatory behavior and decay after saturation as seen earlier in Figure 2a for $A_{\text {init }}=1 \times 10^{-5}$ are present for all initial amplitudes, with waves corresponding to higher initial amplitudes exhibiting an earlier quasi-saturation. Figure $2 \mathrm{~b}$ also indicates that traveling crossflow modes with higher initial amplitudes saturate at progressively larger peak amplitudes.

Now the nonlinear analysis is expanded to a range of other wavelengths. Figure $3 \mathrm{a}$ shows the nonlinear evolution of traveling crossflow vortices with $f=1,500 \mathrm{~Hz}$ and $\lambda_{z}=6,8,10$ and $12 \mathrm{~mm}$, respectively. The number of spanwise Fourier modes retained is 50 in the NPSE computations, which is more than necessary to resolve the spanwise variations of the crossflow vortex. The initial amplitudes, defined as the chordwise perturbation velocity as a fraction of the freestream velocity, for all spanwise wavelengths are held fixed at $10^{-5}$. As expected from the results of linear computations discussed earlier, the $6 \mathrm{~mm}$ wave rises in amplitude first and is followed by waves of consecutively larger wavelengths. The highest overall amplitude is reached by the $8 \mathrm{~mm}$ wave, and not the $12 \mathrm{~mm}$ wave corresponding to the highest value of linear $\mathrm{N}$-factor. Due to nonlinear effects, the $12 \mathrm{~mm}$ wave saturates at a lower peak amplitude than the $8 \mathrm{~mm}$ wave. However, the hierarchy based on peak fundamental mode amplitudes is dependent on the initial amplitude of the wave. In particular, when the initial amplitude is lowered to $2.5 \times 10^{-7}$, the $\lambda_{r}=10 \mathrm{~mm}$ mode has the highest peak amplitude as shown in Figure 3b. As the initial amplitude is further lowered, the nonlinear effects become rather weak; and hence, the $12 \mathrm{~mm}$ wave becomes dominant as predicted by the linear theory (Figure 3c).

The results in Figure 3 also show that, in all cases with a significant effect of nonlinearity (i.e., for sufficiently large initial amplitudes), the amplitude of the fundamental mode evolves in an oscillatory manner. The initial evolution up to the first peak is strictly monotonic; and in general, this first peak also represents the global maximum of the fundamental mode amplitude. However, the subsequent evolution of the fundamental amplitude displays oscillatory behavior superimposed on a decaying trend. The oscillations are believed to have been caused by the interaction of the fundamental and mean flow correction modes. Figure $3 \mathrm{~d}$ shows the evolutions of the fundamental and mean flow correction modes for the $1,500 \mathrm{~Hz}, 10 \mathrm{~mm}$ traveling wave with initial amplitude of $10^{-5}$. Their respective oscillations appear to be approximately 90 degrees out of phase. The fundamental mode, of course, initially drives up the mean flow correction. When it becomes sufficiently large, the mean flow correction mode modifies the boundary layer in such a way that the fundamental mode is stabilized and its amplitude starts to drop. As the mean flow correction mode reaches its peak, the rate of decay of the fundamental mode is approximately the largest. Further drop in the fundamental amplitude, in turn, leads to a decrease in the mean flow correction amplitude, which causes the fundamental mode to grow again. And this interactive process repeats itself downstream. An NPSE computation with only the fundamental and the mean flow correction terms retained reproduces exactly the same oscillatory behavior. Additionally, an NPSE computation with one more Fourier mode retained, namely the first harmonic, also produces very similar result with some modifications in modal amplitudes only, indicating that the first harmonic is not an active player in the interactive process. This confirms that the interaction of two modes and these two modes alone is responsible for the oscillations. 
To help validate the PSE predictions for nonlinear evolution of the primary, traveling crossflow instabilities, DNS computations were also carried out for $1,500 \mathrm{~Hz}$ modes in the traveling frame of reference discussed in Section II. The DNS solutions are based on a spatial grid of size $981 \times 40 \times 278$ in the streamwise, spanwise and wall-normal directions, respectively. Following Jiang et al., ${ }^{25}$ the $i$-coordinate of the grid is aligned with the constant phase surfaces of the traveling crossflow vortex to reduce the number of points required to resolve the wavy structure of the mode in the chordwise direction. To further save on the computational resources, the chordwise extent of the domain is restricted by choosing an inflow location at $x / c=0.15$, i.e., somewhat farther downstream from the lower branch neutral station domain at $x / c \approx 0.05$. The linear eigenfunction of the $1,500 \mathrm{~Hz}$ traveling mode is imposed at the inflow with an initial amplitude that is equivalent to $A_{\text {init }}=5 \times 10^{-7}$.

The DNS results are compared with the NPSE results in Figure 4, where $n$ denotes the harmonic index. The amplitude evolution of the fundamental mode $(m=1)$ from DNS compares very well with that from NPSE right from the beginning of the DNS domain. However, since the NPSE computation is started far upstream of the in-flow location of the DNS domain, the modes for base flow correction and harmonics are well settled at the DNS in-flow location $(x / c=0.15)$. This is not the case with DNS results, since only the fundamental mode is imposed at the inflow for the DNS computation. Consequently, the base flow correction and the harmonics of the fundamental mode undergo a transient phase downstream of the inflow location. Mode $m=2$ has a very short transient region and equilibrates to its downstream asymptotic behavior within a short distance from the inflow location. The base flow correction and the second harmonic modes ( $m=0$ and 3, respectively) settle down next and, after that, the comparison between NPSE and DNS results is good. The higher harmonics remain in the transient stage throughout the DNS computational domain; however, their amplitudes remain low enough such that they are primarily enslaved to the dominant modes up to $m=3$ and, hence, do not exert a noticeable influence on the evolution of the latter modes.

\section{B. Secondary Instability of traveling crossflow vortex}

In this subsection, the secondary instability of traveling crossflow vortices is analyzed and comparisons among of LSIT, NPSE and DNS results are presented.

\section{B.1. Linear Amplification Characteristics for Primary Waves of Selected Frequency-Wavelength Combinations}

The presence of crossflow vortices creates strong localized shear layers within the boundary layer. Secondary instability modes riding on crossflow vortices can be classified into two major types: ${ }^{2}$ secondary modes associated with the strong wall-normal shear of the modified basic state are known as Y modes and those associated with a strong spanwise shear are known as $\mathrm{Z}$ modes. At times, when mean shears in both directions have comparable contributions to the energy production mechanisms associated with the secondary instability, the resulting modes have a mixed character and, hence, are termed as Y/Z modes. ${ }^{9}$ The secondary instability of the non-stationary crossflow vortex with $\lambda_{z}=8 \mathrm{~mm}$ and $f=1,500 \mathrm{~Hz}$ was discussed in Ref. 9. Here, a larger number of cases with different primary wave initial amplitudes and wavelengths are analyzed to help characterize the range of initial amplitudes below which the secondary instability of traveling crossflow modes becomes relatively insignificant in comparison with the secondary instability of stationary crossflow vortices.

The comparison of secondary instability growths on stationary and traveling crossflow vortices of the same initial amplitude was given in Ref. 9. The main result is reproduced in Figure 5 for an initial primary amplitude of $10^{-5}$. The traveling crossflow vortex is seen to sustain secondary instability $\mathrm{N}$-factors just as large as those for stationary waves even though the saturation primary amplitude of the traveling wave is only about one half that of the peak amplitude of stationary mode (see Figure 2a).

The onset of secondary instability is dependent on the primary wave amplitudes exceeding a threshold level that depends on both the local mean flow and the disturbance parameters. To help characterize the threshold amplitudes required for significant amplification of secondary instabilities, a parametric study is carried out for secondary instability modes for various initial amplitudes of the primary wave. Figure 6a shows the $\mathrm{N}$-factor curves for secondary instability modes that first achieve an $\mathrm{N}$-factor of 10 for each initial amplitude of the traveling crossflow

vortex ranging from $A_{\text {init }}=1.5 \times 10^{-7}$ to $10^{-5}$. The wavelength $\left(\lambda_{r}=8 \mathrm{~mm}\right)$ and frequency $(f=1,500 \mathrm{~Hz})$ of the primary disturbance are the same in each of these cases. At relatively high initial amplitudes (approximately $A_{\text {init }} \geq$ 
$5 \times 10^{-6}$ ), the $\mathrm{Y}$ mode dominates. For example, for $A_{\text {init }}=10^{-5}$, the peak $\mathrm{N}$-factor reached by the $\mathrm{Y}$ mode is approximately 1.6 times the maximum $\mathrm{N}$-factor achieved by the Y/Z mode. As the primary amplitude decreases, the maximum $\mathrm{N}$-factors for both modes drop as expected. However, the $\mathrm{Y}$-mode peak $\mathrm{N}$-factor decreases faster with decreasing primary wave amplitude than the Y/Z-mode $\mathrm{N}$-factor. At primary amplitude of $2.5 \times 10^{-6}$, the $\mathrm{Y}$ mode is approximately 13 and the $\mathrm{Z}$-mode $\mathrm{N}$-factor has already overtaken the former with a peak value of 18 . On further reduction of primary wave amplitude to $5 \times 10^{-7}$, no Y mode is picked up by the secondary instability analysis code, indicating that the Y-mode secondary instability has become substantially weaker. On the other hand, the Y/Z mode still reaches a peak $\mathrm{N}$-factor of approximately 10 .

The exact thresholds for the initial primary amplitude required for the onset of secondary instability cannot be defined without carrying out a systematic and time consuming set of computations over a broad range of primary wave amplitudes and streamwise locations. However, the data shown in Figure 6 reveals that the peak $\mathrm{N}$-factor for the Y mode has reduced to less than 3.3 for $A_{\text {init }} \leq 10^{-6}$ and the peak $\mathrm{N}$-factor of the $\mathrm{Y} / \mathrm{Z}$ mode has fallen below 4.2 for $A_{\text {init }} \leq 1.5 \times 10^{-7}$. The corresponding peak amplitudes of the primary wave are approximately $12 \%$ and $7 \%$, respectively. Thus, a conservative estimate is that, below these primary wave peak amplitudes, there is no significant secondary instability growth for the $8 \mathrm{~mm}$ traveling crossflow mode. For the Y modes shown in Figure 6, the peak frequency ranges from 58 to $67.5 \mathrm{kHz}$, and those for the Y/Z modes from 15 to $20 \mathrm{kHz}$.

A comparison is given next of the initial amplitudes of stationary and non-stationary crossflow modes required to reach $\mathrm{N}$-factor values that are likely to correlate with transition. For a stationary crossflow mode of $\lambda_{z}=8 \mathrm{~mm}$, the computations by Choudhari et al. ${ }^{9}$ had shown that the initial primary amplitude required for a secondary instability mode on a traveling crossflow vortex to reach a given $\mathrm{N}$-factor is much smaller than that required on a stationary crossflow vortex. Of course, because of the stronger receptivity mechanisms for stationary crossflow vortices, ${ }^{26}$ the disparity between initial amplitudes of stationary and non-stationary crossflow modes is expected to be substantially greater in most low-amplitude environments. Thus, the next relevant question becomes how much smaller the traveling mode amplitudes need to be so that they do not play any significant role during the transition of the swept wing boundary layer. Based on the discussion of the threshold amplitudes in the preceding paragraph, this is predicted to happen for the $\lambda_{z}=8 \mathrm{~mm}$ traveling mode when its initial amplitude is approximately two orders of magnitude lower than the initial amplitude of the stationary crossflow mode. Of course, this conclusion only holds within the limited context of the present study; however, it provides useful guidance regarding the dominant mechanism for transition onset.

The secondary instability properties for primary waves of wavelengths other than $\lambda_{z}=8 \mathrm{~mm}$ are considered next. Recall from Figure 3 for $A_{\text {init }} \leq 2.5 \times 10^{-7}$ that the peak amplitude of $6 \mathrm{~mm}$ wave becomes small enough so that it is highly unlikely to sustain any significant secondary instability. On the other hand, the longer waves (10 and $12 \mathrm{~mm}$ ) achieve large enough amplitudes to indicate the potential for significant secondary instabilities at farther downstream locations than the secondary instability modes of the $\lambda_{z}=8 \mathrm{~mm}$ wave. Therefore, secondary instability computations are carried out for the 10 and $12 \mathrm{~mm}$ waves for $A_{\text {init }}=2.5 \times 10^{-7}$. The modes that achieve the highest $\mathrm{N}$ factors in each case are plotted in Figure 6b. For both wavelengths, strong Y/Z-mode secondary instability growth is found. In the case of the $12 \mathrm{~mm}$ wave, there is also a strong Y mode (Figure 6b). However, these $\mathrm{Y}$ modes attain an $\mathrm{N}$-factor of 10 or greater significantly downstream of the mid-chord location, so that they would not be expected to play a role in transition even if the stationary crossflow modes were not a factor. Further reduction of their initial amplitudes pushes the large peak amplitudes downstream and hence delay, but not eliminate, the onset of secondary instability, as shown in Figure 6c for a primary amplitude of $A_{\text {init }}=10^{-7}$. However, what can be said about the thresholds for secondary instability is that, for primary amplitudes below $2.5 \times 10^{-7}$ and $10^{-7}$, no $\mathrm{N}$-factor greater than 3 is reached by any secondary instability mode before $x / c=0.35$ and 0.4 , respectively.

Figures 7 a through $7 \mathrm{~d}$ show the Y/Z-mode eigenfunctions on primary waves with $1,500 \mathrm{~Hz}$ in frequencies and 6 , 8,10 and $12 \mathrm{~mm}$ in spanwise wavelengths at chordwise locations where their respective fundamental amplitudes are largest. The primary waves for the 8,10 and $12 \mathrm{~mm}$ cases all have the same initial amplitude of $2.5 \times 10^{-7}$. However, this amplitude is too small to sustain any secondary instability for the $6 \mathrm{~mm}$ primary wave, therefore, an initial amplitude of $1 \times 10^{-5}$ is used instead for that case. These typical eigenfunction shapes are very similar to those found on stationary primary waves. ${ }^{2}$ 
Figure 8 a through $8 \mathrm{~d}$ show the mean chordwise velocity contours of $1,500 \mathrm{~Hz}$ crossflow instability waves in the traveling frame for spanwise wavelengths of $6,8,10$ and $12 \mathrm{~mm}$ at the peak amplitude streamwise locations, respectively, all with an initial amplitude of $10^{-5}$. The shortest wave $(6 \mathrm{~mm})$ shows only a hint of the turnover feature that is almost ubiquitously present in stationary crossflow vortices, but the structure is not nearly as strong as in the latter case. As the spanwise wavelengths becomes longer, this feature diminishes and is hardly discernible for the longest $12 \mathrm{~mm}$ wave. This may be attributed to the relatively low saturation amplitude of the traveling crossflow vortex as discussed earlier.

\section{B.2. Comparison of LSIT Predictions with NPSE and DNS}

Secondary instability computations using NPSE and DNS are also carried out for comparison with the secondary instability theory for the primary mode with $\lambda_{z}=8 \mathrm{~mm}, f=1,500 \mathrm{~Hz}$, and $A_{\text {init }}=5 \times 10^{-7}$. Computations are performed for a fixed-frequency secondary instability mode with a frequency of $13.5 \mathrm{kHz}$, which is close to the most amplified Y/Z mode in this case. As discussed before, at the relatively low value of $A_{\text {init }}$ for the primary crossflow vortex, the $\mathrm{Y}$ mode of secondary instability is nearly stable, but the $\mathrm{Y} / \mathrm{Z}$ mode is still strong. Furthermore, in the experiments on stationary crossflow vortices, ${ }^{27}$ the $\mathrm{Y}$ mode has been less commonly observed than the secondary instability modes associated with spanwise basic state shear. Therefore, a Y/Z mode is chosen for the DNS analysis. The linear eigenfunction of the $13.5 \mathrm{kHz}$ secondary instability mode is imposed at a streamwise location of $x / c=$ 0.265 , i.e., slightly downstream of its neutral point. The minimum wavelength of this mode in the $i$-direction of the grid in the computational domain is approximately $12.7 \mathrm{~mm}$.

The NPSE ${ }^{18}$ computations use 50 spanwise Fourier modes and 281 wall-normal points. Based on previous experiences, ${ }^{11,23}$ these are sufficient to resolve all relevant scales up to the transition location. The DNS computations use a grid of size $2,184 \times 60 \times 418$ points in streamwise, spanwise and wall-normal directions, respectively. An additional set of DNS computations was performed using a grid that was 1.5 times coarser in both spanwise and wall-normal directions. The amplitude evolution based on the coarser grid agreed well with the fine grid solution presented here, except for weak streamwise oscillations in the region downstream of the main lobe in instability growth rate as a function of $x / c$. The streamwise resolution of the DNS grid corresponds to a minimum of 40 points per fundamental wavelength.

Figure 9a shows the amplitude evolutions of the $13.5 \mathrm{kHz}$ Y/Z-mode secondary instability as computed by three different methods, namely, LSIT, NPSE and DNS. The comparison of NPSE and DNS results, while not perfect, is good. The LSIT method, however, is found to over-predict the instability growth downstream by a significant amount compared to the NPSE and DNS results. The differences that accumulate with distance may be attributed to the different degrees of approximations inherent to each of these methods. The LSIT method assumes a quasiparallel base flow (i.e., slowly varying laminar boundary layer as well as traveling crossflow amplitude), which becomes less accurate when the base flow changes relatively rapidly, e.g., in regions of stronger growth or decay of the primary wave. The NPSE method takes into account the non-parallelism of the base flow, but assumes that streamwise second derivatives of perturbation amplitudes are negligible due to their slow variation in the streamwise direction. Furthermore, for numerical stability, the streamwise first derivative of pressure amplitude is neglected (note that the neglected first derivative is that of the slow-varying pressure amplitude, and not that of the overall pressure perturbation) ${ }^{18}$

An equivalent but perhaps more revealing comparison between the LSIT, NPSE and DNS predictions can be made by comparing the corresponding growth rates based on the peak chordwise velocity amplitude of the secondary instability mode (Figure $9 b$ ). In the main region of secondary mode amplification $(x / c<0.37$ ), all three predictions are close to each to other. To our knowledge, this result represents the first DNS based validation of secondary instability theory for traveling crossflow modes. The differences between the NPSE and DNS results within the above region are somewhat smaller than those relative to the LSIT predictions, except in the neighborhood of the peak growth rate location of the secondary mode. Therefore, the cause for the over-prediction of secondary instability growth by the LSIT method in this particular case may be related to the quasi-parallel assumption inherent to LSIT. However, this trend is certainly different from the analogous findings for the secondary instability of stationary crossflow modes. ${ }^{9}$ Furthermore, the effects of a non-parallel basic state also tend to be destabilizing in the linear stability analysis of primary waves. Again, in this particular case, at least, an 
opposite trend appears to hold for the secondary instability evolution. Figure $9 \mathrm{~b}$ also shows significant differences among the three different predictions for chordwise locations beyond the main region of secondary amplification $(x / c>0.37)$. The DNS prediction actually falls in between the LSIT and NPSE predictions.

\section{B.3. Weakly Nonlinear Effects on Secondary Mode Amplification}

The growth and breakdown of secondary instability eventually lead to turbulence. In this process, as the amplitude of the secondary instability becomes progressively larger, its growth rate starts to deviate from that predicted by the linear theory due to the onset of nonlinear effects. Figure 10a shows the effect of initial secondary wave amplitude on the streamwise amplification of the fundamental secondary instability mode of $\mathrm{Y} / \mathrm{Z}$ type and $f=$ $13,500 \mathrm{~Hz}$ corresponding to a finite amplitude primary crossflow disturbance with $\lambda_{z}=8 \mathrm{~mm}$ and $f=1,500 \mathrm{~Hz}$. The initial amplitude of the primary wave is fixed at $5 \times 10^{-7}$ while the initial secondary wave amplitude is varied from $10^{-5}$ to $10^{-3}$. In each case, the secondary wave amplitude is normalized by its initial value, so that the ordinate in Figure 10a corresponds to the growth factor relative to the inflow location. For the smallest secondary initial amplitude $\left(A_{\text {init }}=10^{-5}\right)$, the fundamental amplitude remains sufficiently small throughout the computational domain such that the instability growth may be considered to be linear. As the initial amplitude is increased, the amplitude growth eventually deviates from the linear theory. This occurs near $x / c \approx 0.44$ and 0.34 for $A_{\text {init }}=10^{-4}$ and $10^{-3}$, respectively; and in both cases, the fundamental mode amplitude is approximately $4 \%$ at the respective locations. The corresponding evolution of the mean wall shear in all three cases is shown in Figure 10b. For larger initial amplitudes, the wall shear is higher. For largest initial amplitude, $10^{-3}$, the wall shear begins a relatively rapid rise at $x / c=0.36$, which may be indicative of the onset of transition.

The DNS result for secondary instability at larger initial amplitudes is next compared with the NPSE result along with the essentially linear DNS result of a much smaller initial amplitude. Figure 11 shows the amplitude curves for the fundamental frequency of the $13.5 \mathrm{kHz} \mathrm{Y/Z}$ mode. The amplitudes are normalized with their respective initial values so that all curves initially collapse onto each other. As amplitudes increase, the DNS curve with the larger initial amplitude starts to deviate from curve representing linear amplification due to nonlinear effects. The result of NPSE computation agrees well with the DNS result. The actual peak amplitude reached during the nonlinear evolution is approximately $10 \%$, but no transition is observed before the end of the computational domain. However, the comparison confirms that NPSE should produce good results up to at least this amplitude, which should enable the NPSE method to be used with confidence in similar cases with much less demand on computational resources than DNS.

\section{Concluding Remarks}

This paper extended the previously reported computational studies of swept wing transition due to stationary crossflow instability by considering when traveling crossflow modes might dominate the transition process. As discussed in Ref. 9, the traveling crossflow vortices over swept wing boundary layers support secondary instabilities in much the same way as the stationary crossflow modes for comparable initial amplitudes of the traveling and stationary crossflow vortices. The traveling crossflow vortices saturate at much lower amplitudes than their stationary counterparts, approximately half the saturation amplitude for a stationary crossflow vortex. However, the growth of secondary instabilities sustained by the finite amplitude traveling crossflow vortices is at least as strong as the secondary modes of the stationary crossflow vortices.

The primary traveling crossflow vortex has a higher initial growth rate than the stationary mode, but saturates at significantly lower amplitudes. Additionally, the cross-plane velocity contours for the traveling mode case seem to lack the strong overturning structure of their stationary counterpart when viewed within an appropriately moving coordinate system. Two dominant modes of secondary instability are found for the swept wing configuration investigated herein. The Y mode is dominant at large primary wave amplitudes, but its growth rates diminish faster than that of the Y/Z mode when the primary amplitude decreases. At smaller primary amplitudes of a $\lambda_{z}=8 \mathrm{~mm}$ traveling mode, the $\mathrm{Y}$ mode of secondary instability disappears all together, leaving a single, strongly amplifying Y/Z mode. The critical initial amplitudes enabling the $8 \mathrm{~mm}$ primary wave to sustain significant secondary amplification (i.e., secondary $\mathrm{N}$-factors in excess of 4) have also been estimated for the swept wing configuration of interest. At lower initial amplitudes of the (primary) traveling crossflow modes, the dominant growth of secondary instability shifts to primary modes of larger spanwise wavelengths $\left(\lambda_{z}=10 \mathrm{~mm}\right.$ and $12 \mathrm{~mm}$, respectively). However, large $\mathrm{N}$-factor values typical of those associated with the onset of transition in low disturbance environments are only achieved at far downstream locations, such that transition would appear more likely to occur 
as a result of stationary crossflow modes when the initial amplitudes of the traveling crossflow modes have reduced to these levels. Transition scenarios associated with the joint development of stationary and traveling crossflow modes will be investigated in future work.

DNS computations are carried out for a Y/Z-mode secondary instability of $13.5 \mathrm{kHz}$ riding a $1,500 \mathrm{~Hz}$ traveling crossflow vortex of $8 \mathrm{~mm}$ in spanwise wavelength. Comparisons with NPSE and LSIT results show that linear secondary instability over predict the growth of secondary instability in this particular case and the reason is likely to be non-parallel effects of the base flow.

\section{Acknowledgments}

This work was performed as part of the Revolutionary Computational Aerosciences (RCA) discipline under the Aeronautical Sciences (AS) project of NASA's Fundamental Aeronautics Program. The authors would like to thank Prof. Pino Martin of the University of Maryland for providing the original version of the DNS code used in this effort.

\section{References}

${ }^{1}$ Poll, D. I. A., "Transition in the Infinite Swept Attachment Line Boundary Layer," The Aeron. Quarterly, Vol. 30,1979, pp. 607-629.

${ }^{2}$ Malik, M. R., Li, F., Choudhari, M., and Chang, C.-L., "Secondary Instability of Crossflow Vortices and SweptWing Boundary-Layer Transition,” J. Fluid Mech., Vol. 399, 1999, pp. 85-115.

${ }^{3}$ Kohama, Y., Saric, W. S., and Hoos, J. A., "A high-frequency secondary instability of crossflow vortices that leads to transition," in Boundary Layer Transition and Control, Proc. Royal Aeronautical Society, Cambridge, 8-12 April, 1991, London, UK.

${ }^{4}$ Balachandar, S., Streett, C. L., and Malik, M. R., "Secondary Instability in a Rotating Disk Flow," J. Fluid Mech., Vol. 242, 1992, pp. 323-347.

${ }^{5}$ Malik, M. R., Li, F., and Chang, C.-L., "Crossflow disturbances in three-dimensional boundary layers: Nonlinear development, wave interaction and secondary instability," J. Fluid Mech., Vol. 268, 1994, pp. 1-36.

${ }^{6}$ Wassermann, P. and Kloker, M., "Mechanisms and Passive Control of Crossflow-Vortex-Induced Transition in a Three-Dimensional Boundary Layer," J. Fluid Mech., Vol. 456, 2002, pp. 49-84.

${ }^{7}$ Bippes, H., "Basic experiments on transition in three-dimensional boundary layers dominated by crossflow instability," Progress in Aerospace Sciences, Vol. 35, 1999, pp. 363-412.

${ }^{8}$ Wassermann, P. and Kloker, M., "Transition Mechanisms Induced by Traveling Crossflow Vortices in a ThreeDimensional Boundary Layer," J. Fluid Mech., Vol. 483, 2003, pp. 67-89.

9 Choudhari, M., Li, F., Duan, L., Carpenter, M. H., Streett, C. L., and Malik, M. R., "Towards Bridging the Gap in Hoslistic Transtion Prediction via Numerical Simulations," AIAA Paper 2013-2718, 2013.

${ }^{10}$ Down, R. S. and White, E. B., "Free-stream Turbulence and Development of Crossflow Disturbances," J. Fluid Mech. Vol. 735, 2013, pp. 347-380.

${ }^{11}$ Li, F. and Choudhari, M., "Spatially Developing Secondary Instabilities and Attachment Line Instability in Supersonic Boundary Layers," AIAA Paper 2008-0590, 2008.

${ }_{12}$ Malik, M. R., Li, F., and Chang, C.-L., "Nonlinear Crossflow Disturbances and Secondary Instability in ThreeDimensional Boundary Layers," Proc. IUTAM Symp., Kluwer Acad. Publ., pp 257-266, 1996.

${ }^{13}$ Lin, R. S. and Malik, M. R., "On the Stability of Attachment-line Boundary Layers. Part I. The Incompressible Swept Hiemenz Flow," J. Fluid Mech. Vol. 311, 1996, pp. 239-255.

${ }^{14}$ Herbert, T., "Secondary Instability of Boundary Layers,", Ann. Rev. Fluid Mech. Vol. 20, 1988, pp. 487-526.

${ }^{15}$ Belisle, M., Neale, T., Reed, H., and Saric, W. "Design of a Swept-Wing Laminar Flow Control Flight

Experiment for Transonic Aircraft," AIAA paper 2010-4381, 2010.

${ }^{16}$ Wie, Y.-S., "BLSTA - A Boundary Layer Code for Stability Analysis," NASA CR 4481, 1992.

${ }^{17}$ Saric, W. S., "Flight Experiments on Swept-Wing Roughness Receptivity: Phase 2. Feasibility of a laminar flow flight test," Phase 2, Year 1 Draft Report for AFOSR Grant FA955008-1-0475, June, 2009.

${ }^{18}$ Chang, C.-L., "Langley Stability and Transition Analysis Code (LASTRAC) Version 1.2 User Manual," NASA/TM-2004-213233, June, 2004. 
${ }^{19}$ Martin, M. P., Taylor, E. M., Wu, M., and Weirs, V. G., "A Bandwidth-Optimized WENO Scheme for Effective Direct Numerical Simulations of Compressible Turbulence," J. Comp. Phys., Vol. 220, 2006, pp. 270-298.

${ }^{20}$ Duan, L., Choudhari, M., and Li, F., "Direct Numerical Simulation of Crossflow-Induced Transition in a Swept Wing Boundary Layer," AIAA Paper 2013-2617, 2013.

${ }^{21}$ Choudhari, M., Chang, C.-L., Streett, C. L. and Balakumar, P., "Integrated Transition Prediction: A Case Study in Supersonic Laminar Flow Control," AIAA Paper 2003-0973, 2003.

${ }^{22}$ Chang, C.-L. and Choudhari, M. M., "Boundary-Layer Receptivity and Integrated Transition Prediction," AIAA Paper 2005-0526, 2005.

${ }^{23}$ Li, F., Choudhari, M., Chang, C.-L., Streett, C. L., and Carpenter, M. H., "Roughness Based Crossflow Transition Control: A Computational Assessment," AIAA Paper 2009-4105, 2009.

${ }^{24}$ Malik, M.R., Liao, W., Li, F., and Choudhari, M., "DRE-Enhanced Swept-Wing Natural Laminar Flow at High Reynolds Numbers," AIAA Paper 2013-412, 2013.

${ }^{25}$ Jiang, L., Choudhari M., Chang, C., and Liu, C., "Direct Numerical Simulations of Crossflow Disturbances in Supersonic Boundary Layers," AIAA Paper 2004-589, 2004.

${ }^{26}$ Choudhari, M., "Roughness-Induced Generation of Crossflow Vortices in Three-Dimensional Boundary Layers," Theor. and Comp. Fluid Dyn., Vol. 5, Feb. 1994, pp. 1-31.

${ }^{27}$ White E. B. and Saric W. S., "Secondary Instabilty of Crossflow Vortices," J. Fluid Mech. Vol. 525, 2005, pp. 275-308. 


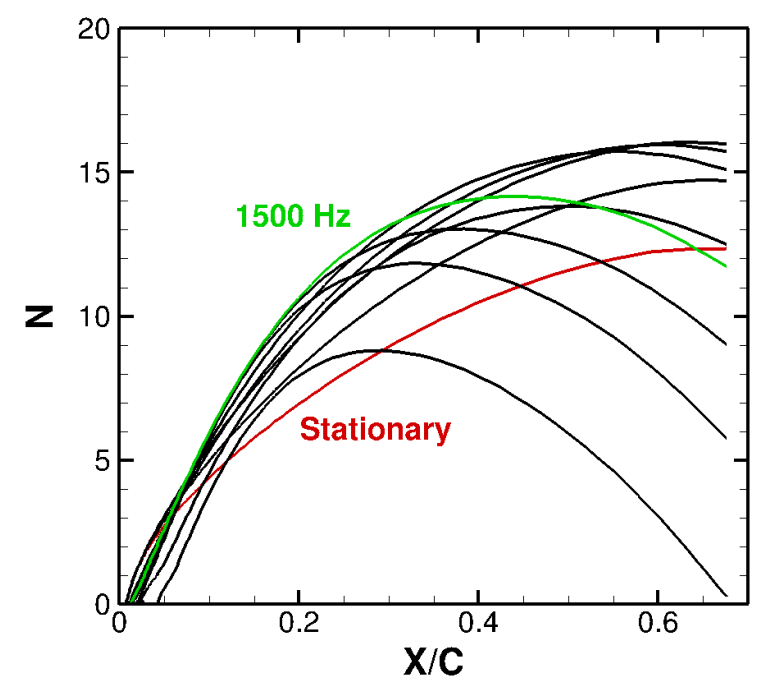

a) Crossflow instability modes with $\lambda_{L}=8 \mathrm{~mm}$ and frequency ranging from 0 to $2.25 \mathrm{kHz}$. The $\mathrm{N}$-factor first reaches a value of 10 for the $1,500 \mathrm{~Hz}$ mode indicated by green curve. $\mathrm{N}$-factor curve for the stationary crossflow mode is shown in red

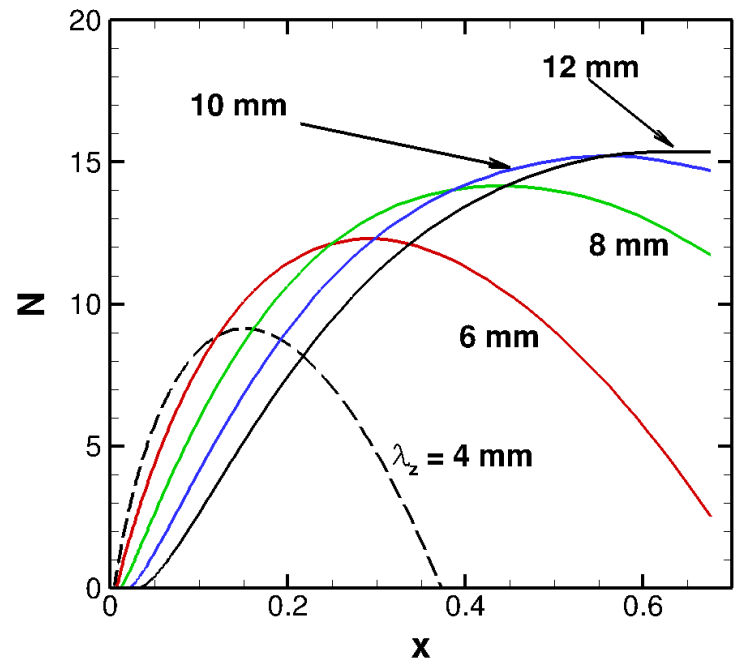

b) Linear $\mathrm{N}$-factors for various spanwise wavelengths at a frequency of $1.5 \mathrm{kHz}$.

Figure 1. Linear N-factors for crossflow instability.

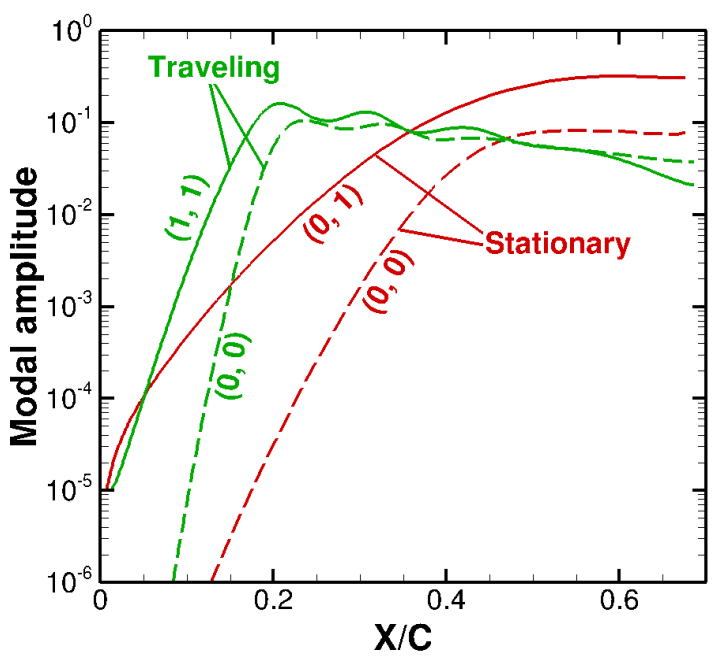

a) Stationary and traveling crossflow ( $f=1.5 \mathrm{kHz}$ ) modes with $\lambda=8 \mathrm{~mm}$ and the same initial amplitude $\left(A_{\text {init }}=1 \times 10^{-5}\right)$ : Comparison of modal amplitudes for the fundamental and mean flow correction modes.

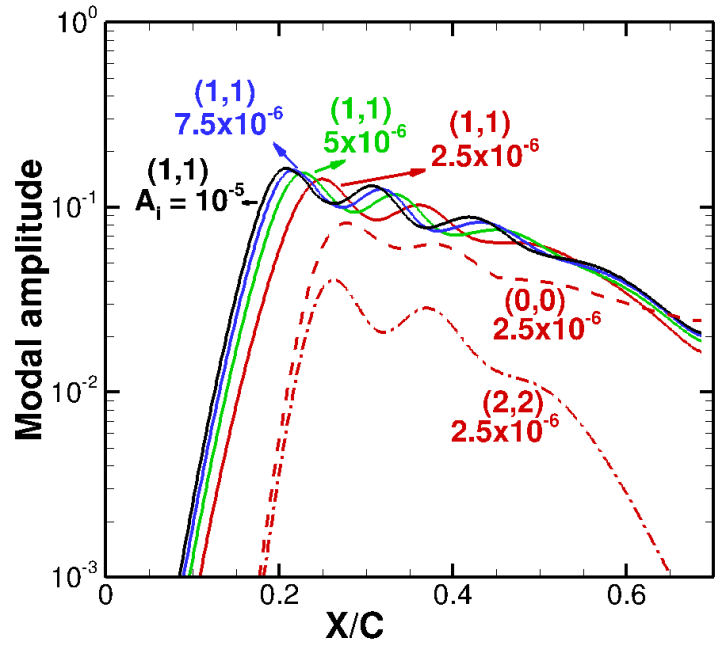

b) Fundamental amplitude for $8 \mathrm{~mm}$ traveling crossflow vortices with different initial amplitudes. The amplitudes of mean flow correction and the first harmonic are also shown for the case with the smallest initial amplitude $\left(A_{\text {init }}=2.5 \times 10^{-6}\right)$.

Figure 2. Nonlinear evolution of crossflow vortex modal amplitudes (Ref. 9). 


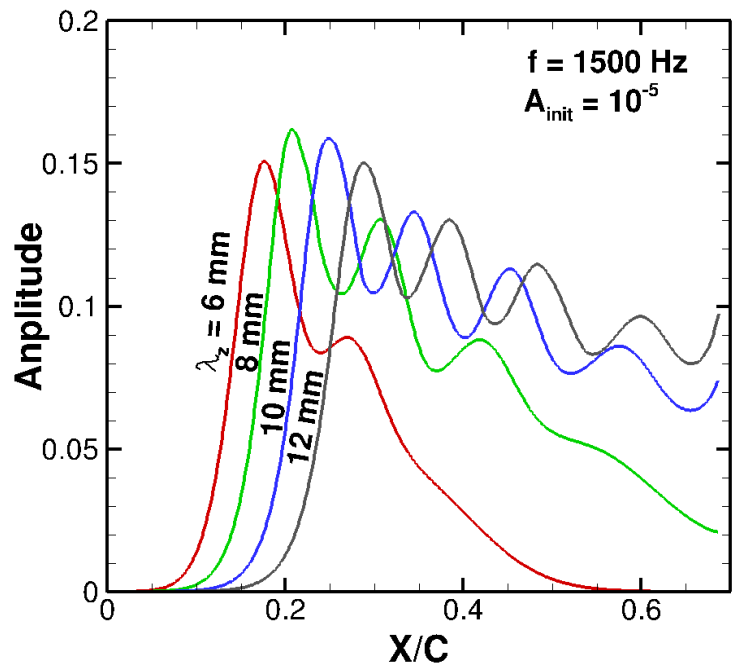

a) Fundamental amplitudes. $f=1.5 \mathrm{kHz}$, $A_{\text {init }}=10^{-5}$.

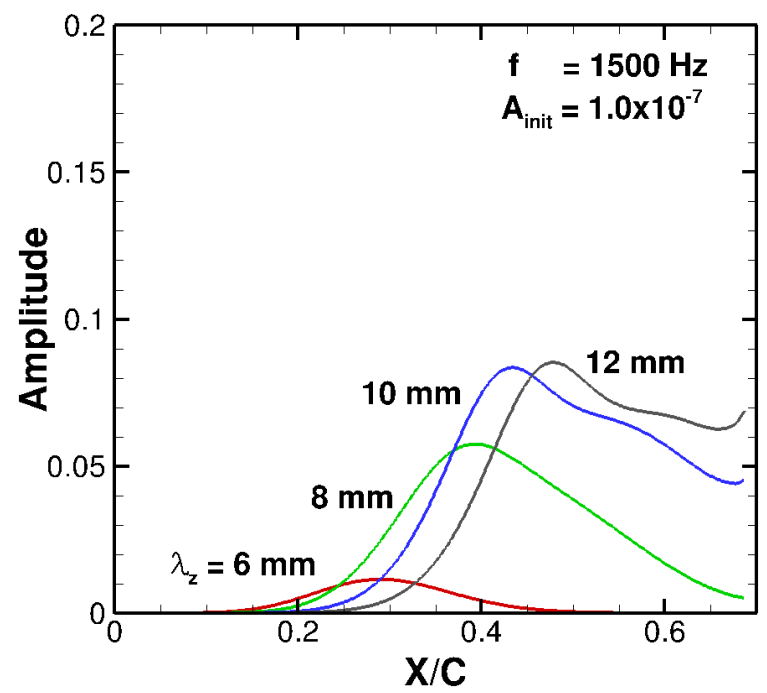

c) Fundamental amplitudes. $f=1.5 \mathrm{kHz}$, $A_{\text {init }}=1 \times 10^{7}$.

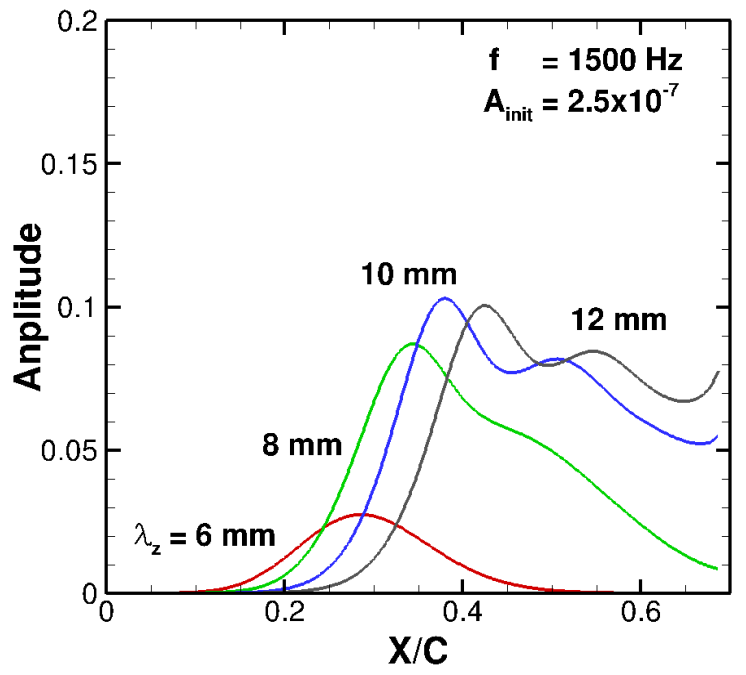

b) Fundamental amplitudes. $f=1.5 \mathrm{kHz}$,

$$
A_{\text {init }}=2.5 \times 10^{-7} \text {. }
$$

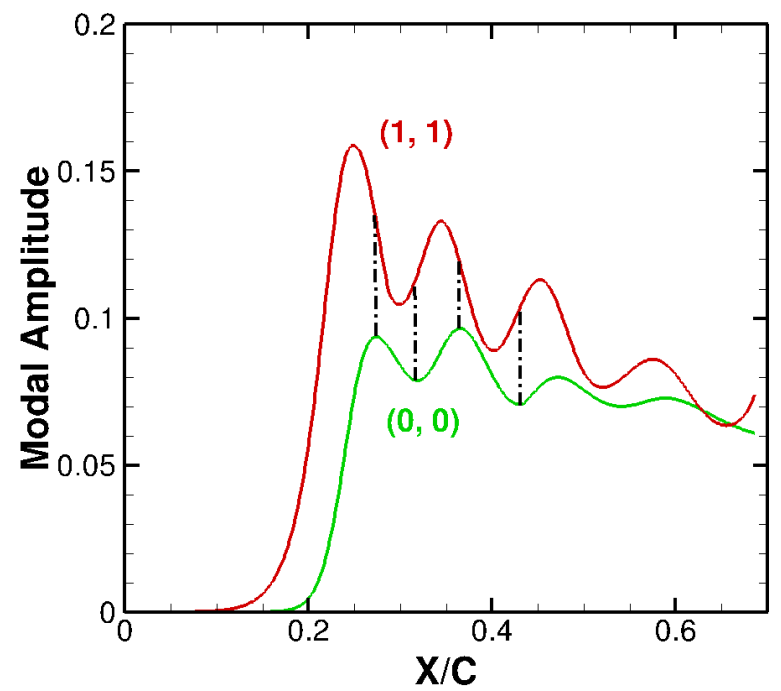

d) Fundamental and mean flow correction modes with approximately 90 degrees phase difference. Vertical dash-dot lines indicate corresponding locations of peak meanflow correction and rapid rate of change of fundamental mode. $A_{\text {init }}=10^{-5}, \lambda_{\mathrm{z}}$ $=10 \mathrm{~mm}, f=1.5 \mathrm{kHz}$.

Figure 3. Evolution of modal amplitudes 


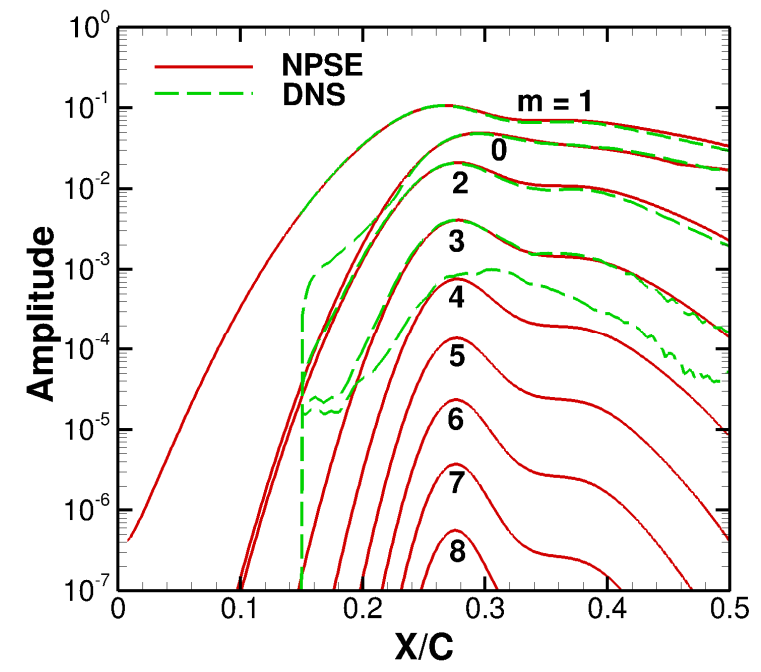

Figure 4. Evolution of modal amplitudes for traveling crossflow vortices with initial amplitude of $5 \times 10^{-7}$. Comparison between results of nonlinear PSE and direct numerical simulations. Solid red lines and dashed green lines present results of NPSE and DNS computations, respectively.

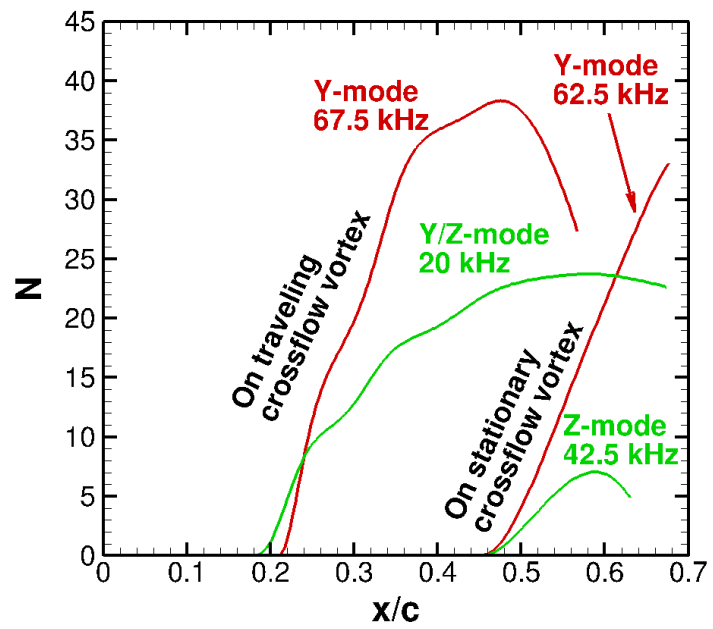

Figure 5. Comparison of $\mathrm{N}$-factor curves for most dangerous secondary instability modes of stationary and traveling crossflow vortices. The metric for growth potential corresponds to earliest attainment of $\mathbf{N}=10$, or of the highest $\mathrm{N}$-factor if $\mathrm{N}=10$ is never achieved. Both stationary and traveling crossflow vortices havethe same spanwise wavelength of $8 \mathrm{~mm}$, and the traveling mode has a frequency of $1,500 \mathrm{~Hz}$ (Ref. 9). 


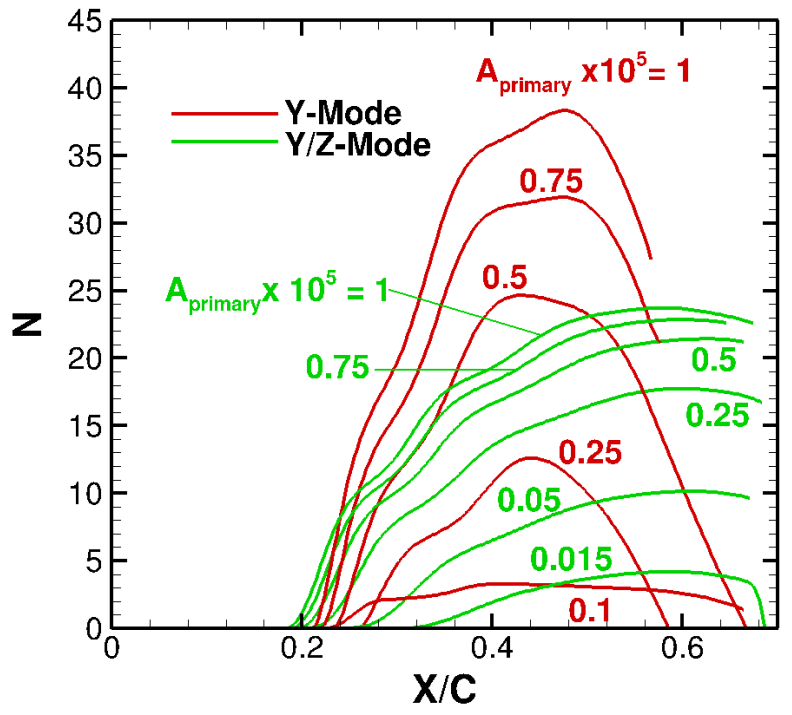

a) Effect of different primary wave initial amplitudes, $\lambda_{z}=8 \mathrm{~mm}$

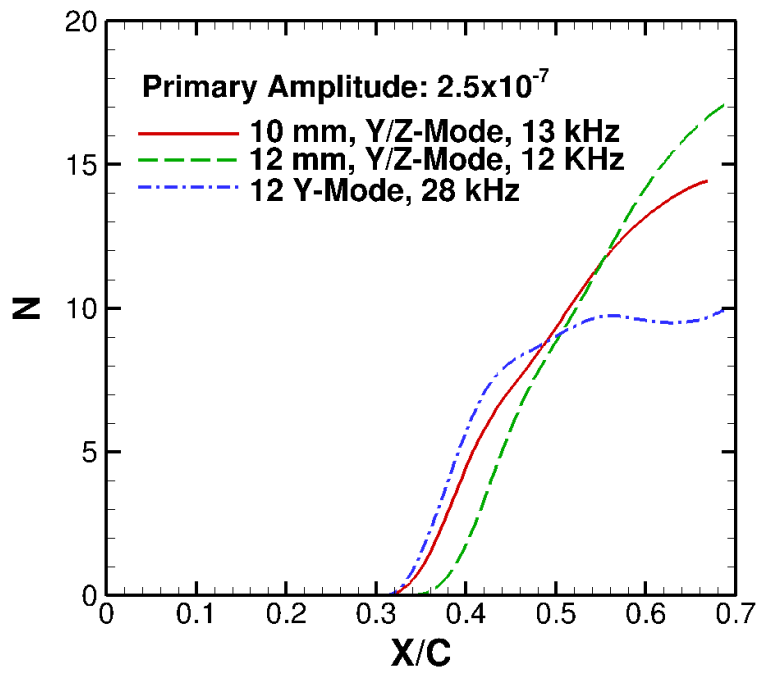

b) $\lambda_{z}=10$ and $12 \mathrm{~mm}, A_{\text {init }}=2.5 \times 10^{-7}$.

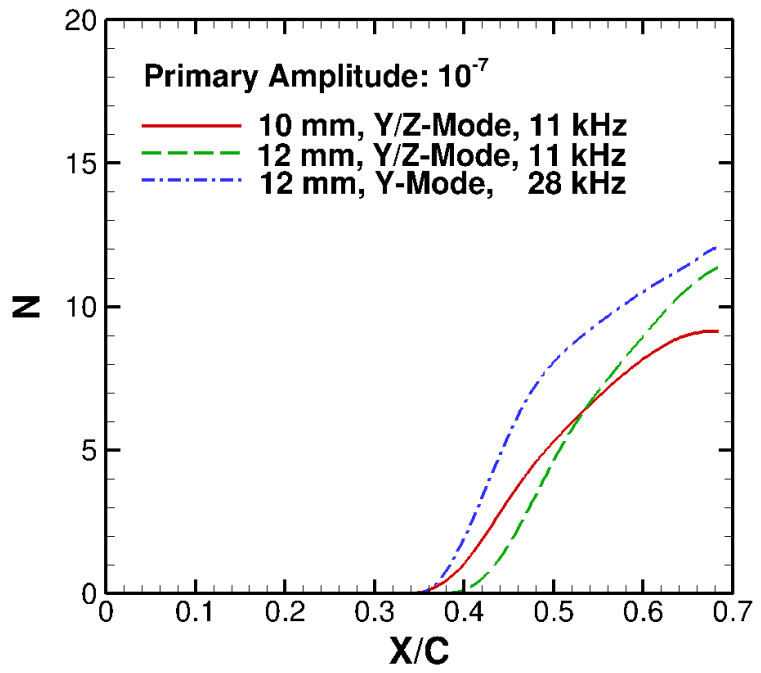

c) $\lambda_{\mathrm{z}}=10$ and $12 \mathrm{~mm}, A_{\text {init }}=10^{-7}$.

Figure 6. Peak N-factors of secondary instability of traveling crossflow vortices. 


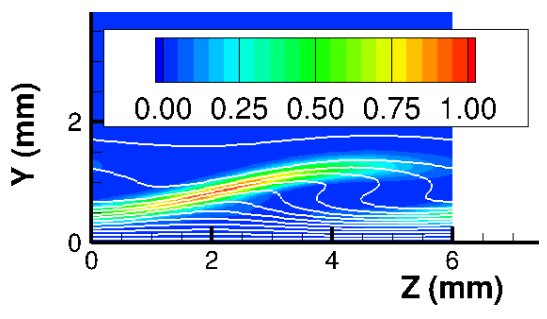

a) $x / c=0.18, \lambda_{z}=6 \mathrm{~mm}$

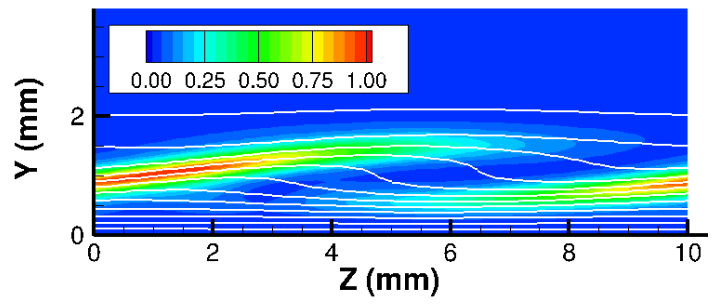

c) $x / c=0.38, \lambda_{z}=10 \mathrm{~mm}$

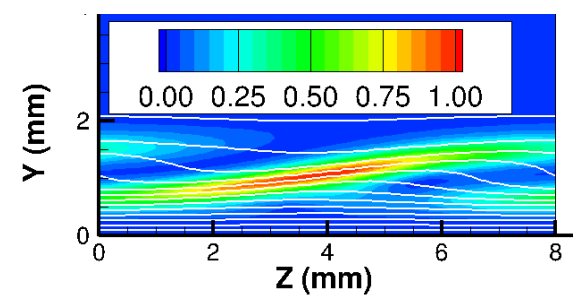

b) $x / c=0.21, \lambda_{z}=8 \mathrm{~mm}$

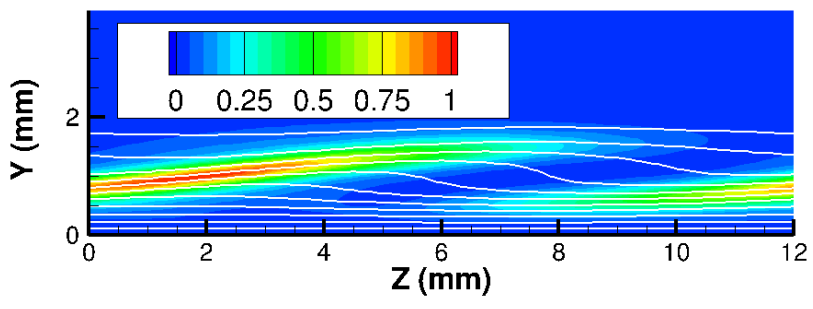

d) $x / c=0.43, \lambda_{z}=12 \mathrm{~mm}$

Figure 7. Y/Z mode eigenfunctions (chordwise perturbation velocity) for secondary instability modes on primary waves with $f=1500 \mathrm{~Hz}$ and varying spanwise wavelength. Flood contours represent chordwise velocity perturbation associated with the secondray instability; white lines represent the base flow chordwise velocity.

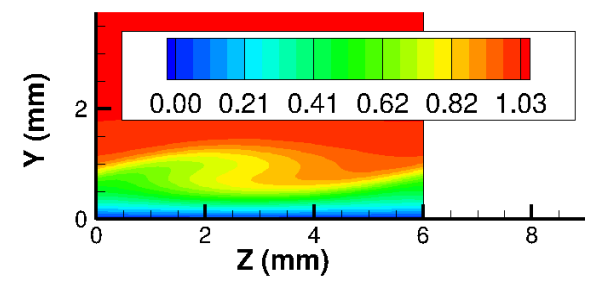

a) $x / c=0.18 . \lambda_{z}=6 \mathrm{~mm}$

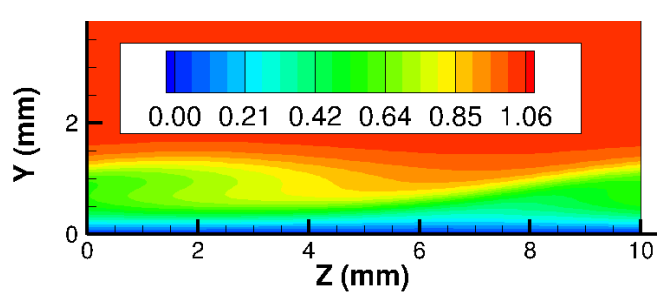

c) $x / c=0.25, \lambda_{z}=10 \mathrm{~mm}$

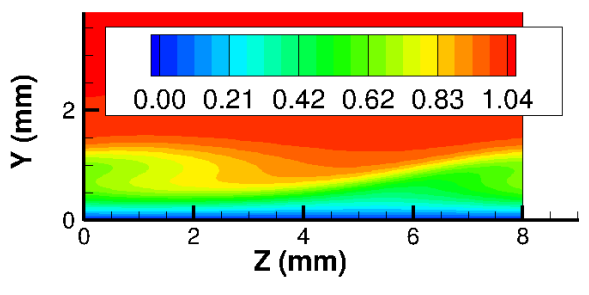

b) $x / c=0.21, \lambda_{z}=8 \mathrm{~mm}$

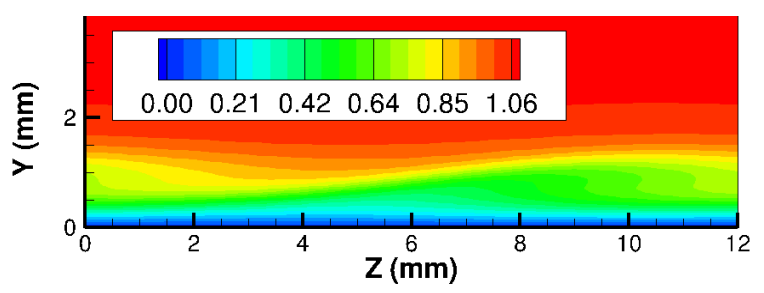

c) $x / c=0.29, \lambda_{z}=12 \mathrm{~mm}$

Figure 8. Chordwise velocity $\left(u / U_{\infty}\right)$ contours associated with finite amplitude traveling crossflow modes of various spanwsie wavelengths at chordwise location corresponding to the maximum wave amplitude in that case ( $A_{\text {init }}=10^{-5}$ in each case). 


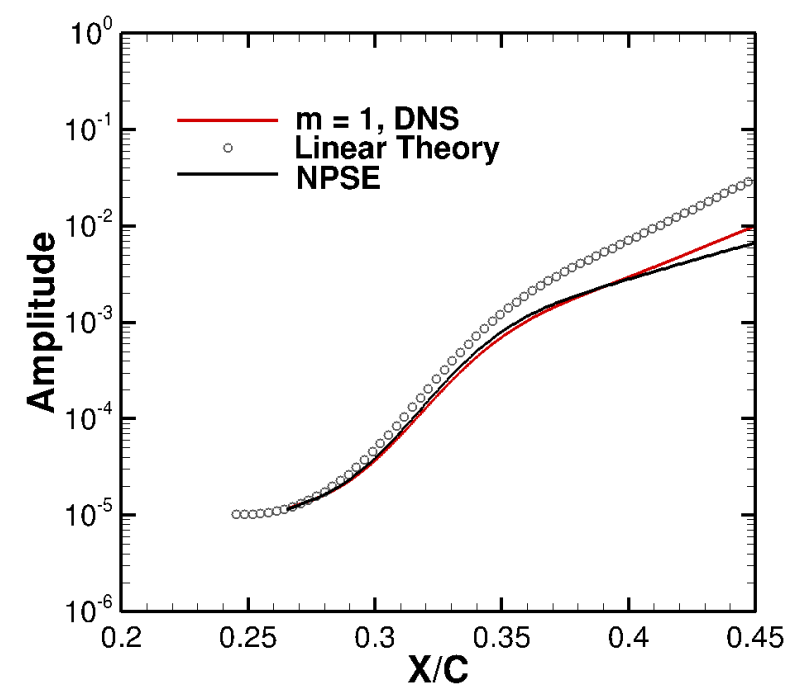

a) Fundamental amplitude of secondary wave

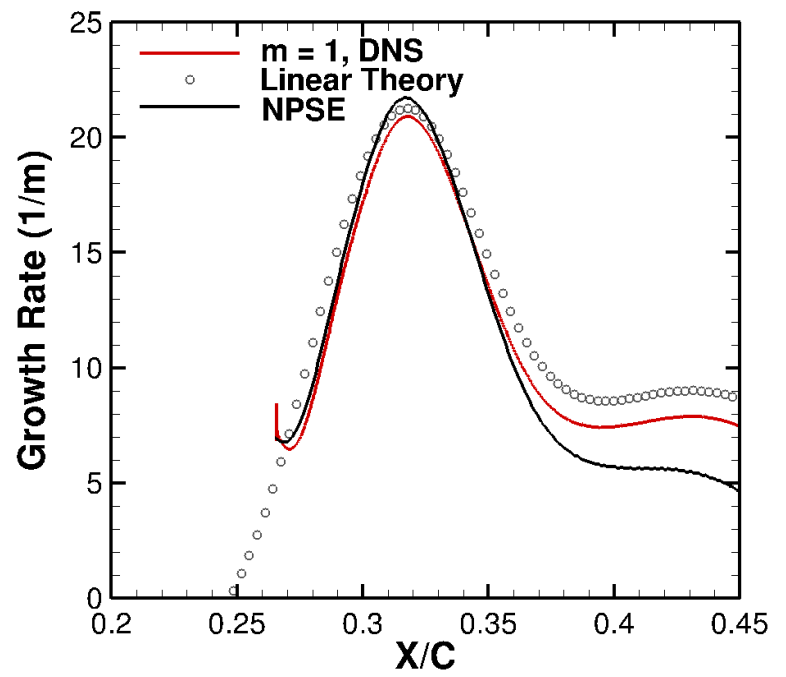

b) Growth rate of secondary wave

Figure 9. Comparison of LSIT, NPSE, and DNS predictions for secondary wave evolution (primary wave: $\lambda_{z}=$ $8 \mathrm{~mm}, f=1500 \mathrm{~Hz}$; secondary wave: $f=13.5 \mathrm{kHz}, A_{\text {init }}=5 \times 10^{-7}$ ).

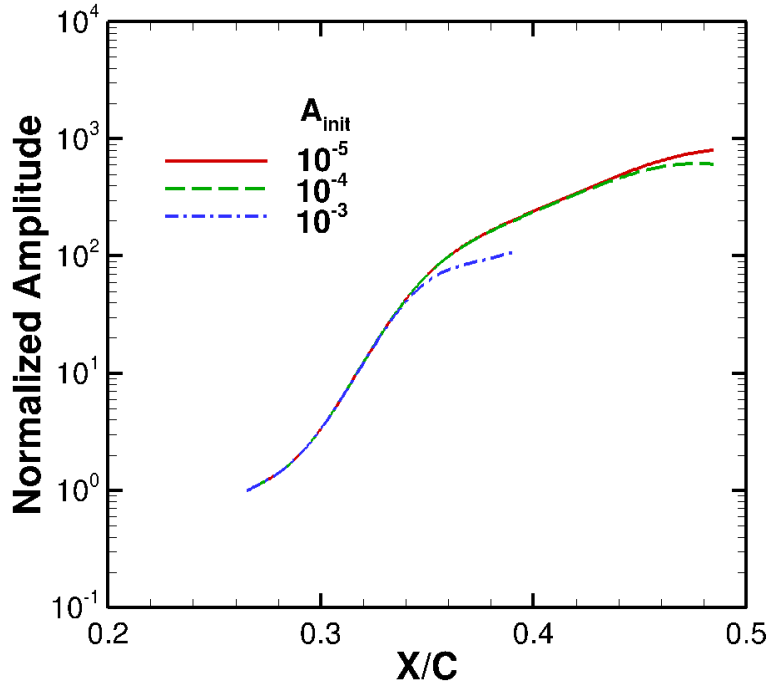

a) Amplitudes.

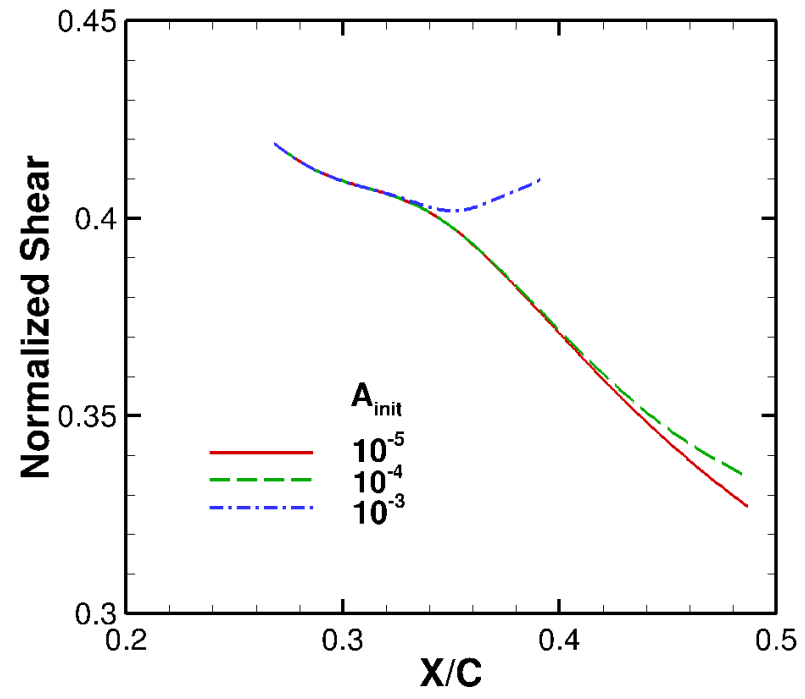

b) Mean wall shear.

Figure 10. Nonlinear evolution for selected initial amplitudes of the secondary wave. 


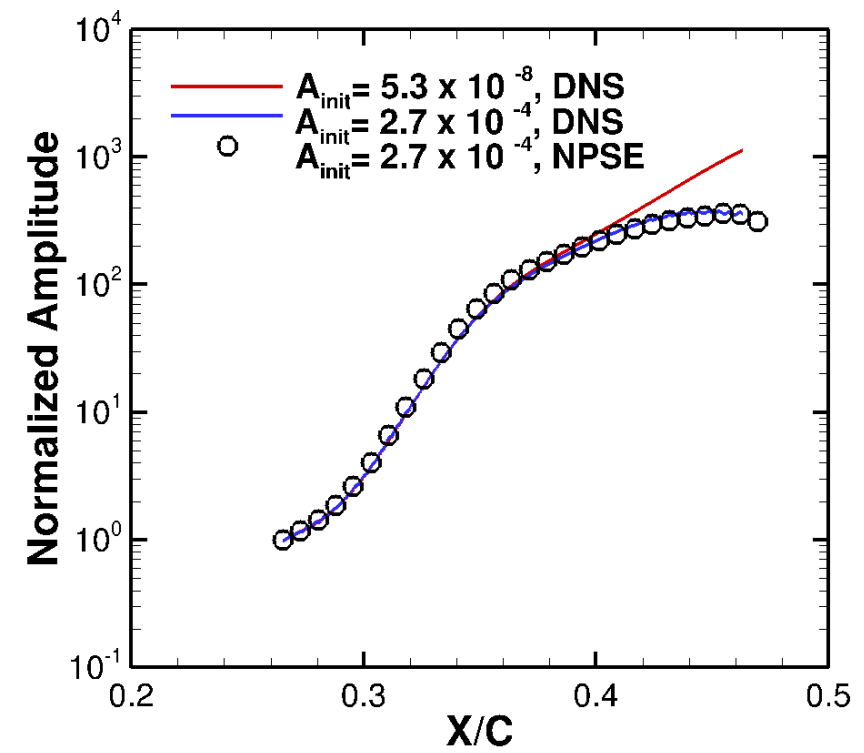

Figure 11. Chordwise evolution of fundamental secondary mode for different initial amplitudes: Comparison between NPSE and DNS. 